\title{
The Temperature-Dependent Nature of Coronal Dimmings
}

\author{
Eva Robbrecht ${ }^{1}$ and Yi-Ming Wang ${ }^{2}$ \\ Eva.Robbrecht@oma.be, Yi.Wang@nrl.navy.mil
}

\begin{abstract}
The opening-up of the magnetic field during solar eruptive events is often accompanied by a dimming of the local coronal emission. From observations of filament eruptions recorded with the Extreme-Ultraviolet Imager on STEREO during 2008-2009, it is evident that these dimmings are much more pronounced in $19.5 \mathrm{~nm}$ than in the lower-temperature line $17.1 \mathrm{~nm}$, as viewed either on the disk or above the limb. We conclude that most of the cooler coronal plasma is not ejected but remains gravitationally bound when the loops open up. This result is consistent with Doppler measurements by Imada and coworkers, who found that the upflow speeds in a transient coronal hole increased dramatically above a temperature of $1 \mathrm{MK}$; it is also consistent with the quasistatic behavior of polar plumes, as compared with the hotter interplume regions that are the main source of the fast solar wind. When the open flux reconnects and closes down again, the trapped plasma is initially heated to such high temperatures that it is no longer visible at Fe IX $17.1 \mathrm{~nm}$. Correspondingly, $17.1 \mathrm{~nm}$ images show a dark ribbon or "heat wave" propagating away from the polarity inversion line and coinciding with the brightened Fe XV $28.4 \mathrm{~nm}$ and Fe XII $19.5 \mathrm{~nm}$ post-eruptive loops and their footpoint areas. Such dark ribbons provide a clear example of dimmings that are not caused by a density depletion. The propagation of the "heat wave" is driven by the closing-down, not the opening-up, of flux and can be observed both off-limb and on-disk.
\end{abstract}

Subject headings: Sun: corona - Sun: coronal mass ejections (CMEs) - Sun: filaments, prominences — Sun: magnetic topology — solar wind — Sun: UV radiation

\footnotetext{
${ }^{1}$ Royal Observatory of Belgium, Ringlaan 3 - 1180 Brussels, Belgium

${ }^{2}$ Naval Research Laboratory, 4555 Overlook Ave SW, Washington, DC 20375-5352, USA
} 


\section{Introduction}

Filaments eruptions and other ejections of mass from the Sun are often accompanied by a dimming of the local coronal emission at many different wavelengths, and by the formation of transient coronal holes (see, e.g., Harrison \& Lyons 2000; Kahler \& Hudson 2001; Harrison et al. 2003; Howard \& Harrison 2004; Attrill et al. 2006; Harra et al. 2007; Imada et al. 2007; Reinard \& Biesecker 2008, 2009; Jin et al. 2009; Dai et al. 2010). The dimmings are in most cases caused by a decrease in the coronal density due to the opening-up of the magnetic field and escape of the entrained material into the heliosphere. The closing-down of the flux proceeds from the inside outward, with the field lines rooted nearest the photospheric polarity inversion line (PIL) pinching off first, giving rise to a progressively growing posteruption loop arcade (Kopp \& Pneumann 1976). Chromospheric evaporation fills each newly reconnected loop with high-temperature plasma, which cools as the loop collapses; thus the hottest loops are located at the leading edge of the outward-expanding "reconnection wave" (see, e.g., Warren et al. 1999; Sheeley et al. 2004, 2007).

The different viewing angles afforded by the two STEREO spacecraft (Howard et al. 2008) provide a unique opportunity to study the 3-dimensional structure of coronal dimmings and post-eruption arcades. Moreover, with the Extreme-Ultraviolet Imager (EUVI), these events can be observed simultaneously in the 17.1, 19.5, and $28.4 \mathrm{~nm}$ bandpasses, corresponding to temperatures of $\sim 1, \sim 1.5$ and $\sim 2$ MK respectively, thereby allowing one to distinguish more easily between "real" dimmings due to mass loss (often termed transient coronal holes) and dimmings due to heating and cooling effects in post-eruption arcades. We have studied several events involving the eruption of high-latitude filaments during the 2008-2009 activity minimum, when the separation between the STEREO A and B spacecraft was on the order of $90^{\circ}$. In this Letter, we focus on new results concerning the temperature dependence of the dimmings and the subsequent reconnection waves.

\section{EUV Observations}

The sequence of images in Figure 1 shows a filament eruption at the northeast limb on 2009 January 14, as observed by EUVI A in the 17.1, 19.5, and $28.4 \mathrm{~nm}$ bandpasses (see also the accompanying online movies). Each image represents the ratio of the local brightness at the given time to that at 19:06 UT on January 13, before the start of the eruption; a shift has been applied to remove the effect of the photospheric differential rotation on the disk (Howard, Harvey and Forgach, 1990). By taking the ratio of intensities rather than subtracting them, we are able to bring out features above the limb which, because of the rapid falloff of the coronal density with height, would not be visible in ordinary base-difference 
images.

At 04:06 UT, the last of the prominence material (which is best seen in He II $30.4 \mathrm{~nm}$ ) is being ejected from the limb. This dense, cool material is only faintly visible at 19.5 and $28.4 \mathrm{~nm}$, but appears in Fe IX $17.1 \mathrm{~nm}$ as a bright blob with a narrow, dark tail. We interpret this dark tail as a density depletion associated with the pinching-off of the magnetic field behind the ejection. Poleward and equatorward of the disconnection region, however, the corona is noticeably darker in the higher-temperature lines than in $17.1 \mathrm{~nm}$.

At 06:06 UT (second row of images in Figure 1), a cusp-shaped post-eruption arcade has begun to form in 19.5 and $28.4 \mathrm{~nm}$, with the structure being brighter and more extended in the latter wavelength. In both cases, the surrounding corona (off-limb) has been strongly depleted of material at the given temperature, as indicated by the dark voids in the images. In contrast, the $17.1 \mathrm{~nm}$ image is dominated by neutral gray, and shows neither a bright arcade nor a large region of depleted density. Instead, we continue to see a remnant of the wakelike depletion and some dark areas which lie inside the outer boundary of the bright 28.4 arcade (indicated by the yellow contours), and which evidently represent plasma that has been heated to temperatures well above $1 \mathrm{MK}$. The same situation continues to hold as the hot post-eruption arcades expand (bottom row of images).

Figure 2 shows the filament eruption and its aftermath as viewed on the disk from the STEREO B spacecraft. Here, each image represents the ratio of the local brightness at the given time to that recorded $\sim 2 \mathrm{hr}$ earlier (rather than before the eruption). The Fe XII $19.5 \mathrm{~nm}$ image taken at 05:05 UT shows a pair of large, dark transient coronal holes, one on each side of the footpoint brightenings of the post-eruption arcade. It should be noted that the brightenings appear in the vicinity of the PIL well before the holes (which remain essentially stationary) reach their darkest level. The transient holes and footpoint brightenings are barely visible in the Fe IX $17.1 \mathrm{~nm}$ image, as expected from the corresponding limb views of Figure 1, where the $17.1 \mathrm{~nm}$ intensities undergo relatively little change during the event. More puzzling, at first sight, is the rather weak signature of the transient holes at $28.4 \mathrm{~nm}$, despite the very strong darkenings that are seen above the limb in Figure 1. The weakness of the on-disk holes can be attributed to contributions to the $28.4 \mathrm{~nm}$ bandpass from low-temperature lines such as Si VII $27.5 \mathrm{~nm}$, which become particularly significant in darker regions of the disk (see e.g., Figure 21 in Del Zanna et al. 2003).

Between 05:05 and 07:05 UT, the brightenings increase in intensity and continue to spread through the area occupied by the transient holes. In the $17.1 \mathrm{~nm}$ running-ratio image at 07:06 UT, the poleward and (to a lesser extent) the equatorward sides of the footpoint brightenings are bordered by dark ribbons, which lie inside the boundaries of the bright 28.4 and $19.5 \mathrm{~nm}$ emission. These dark ribbons evidently represent the footpoint areas of the 
newly reconnected loops which earlier contained Fe IX plasma but have now been heated to higher temperatures (see Fig. 1). Thus the dark, outward-propagating dark ribbons observed in the relatively cool $17.1 \mathrm{~nm}$ line are a heating effect due to the closing-down of flux, and not a density-depletion effect due to the opening-up of field lines. Note that, in running-ratio images, transient holes no longer appear as dark areas after they have reached their darkest level in base-ratio images; comparing the two types of images thus offers a way to distinguish the "heat waves" from the transient coronal holes.

At 09:05 UT, the footpoint brightenings continue to propagate outward, but no further intensity increases occur between the diverging fronts. A dark ribbon is still present along the limbward side of the poleward-propagating $17.1 \mathrm{~nm}$ brightening. Comparing the positions of the 19.5 and $28.4 \mathrm{~nm}$ brightenings, we see that the higher-latitude fronts appear to be shifted more relative to each other than the equatorward-propagating fronts. This difference may be a projection effect caused by the fact that the $28.4 \mathrm{~nm}$ emission extends to greater heights than the $19.5 \mathrm{~nm}$ emission, and thus seems to extend farther toward the limb.

As another characteristic example, Figures 3 and 4 show the off-limb and on-disk views of a filament eruption that occurred on 2008 December 27 (see also the online movies). The temperature/heating effect is clearly seen in the limb view from EUVI A (Figure 3), where the post-eruption arcade is bright in $28.4 \mathrm{~nm}$, fainter in $19.5 \mathrm{~nm}$, and dark in $17.1 \mathrm{~nm}$. Conversely, the region of strongly depleted $28.4 \mathrm{~nm}$ emission above the arcade appears as neutral gray in the $17.1 \mathrm{~nm}$ base-ratio images, indicating that it is mainly the hotter plasma that escapes when the magnetic field opens up. Considering now the on-disk view from EUVI B (Figure 4), we first note that the dimmings in the $19.5 \mathrm{~nm}$ running-ratio images at 05:06 and 07:06 UT represent transient coronal holes. The faint Y-shaped darkening seen at 05:06 UT in $17.1 \mathrm{~nm}$ is a heating effect, since it coincides with a similarly shaped brightening in $28.4 \mathrm{~nm}$. Subsequently, as this plasma cools, the double-ribbon brightening begins to appear in $17.1 \mathrm{~nm}$, bordered on its poleward and equatorward sides by dark patches that represent the outward-propagating $17.1 \mathrm{~nm}$ "heat wave".

The high-latitude filament eruptions of December 27 and January 14 both gave rise to slow CMEs observed with the white-light coronagraphs on STEREO.

\section{Physical Interpretation}

Figure 5shows the coronal magnetic field on 2009 January 14, as viewed from STEREO B; the field lines were derived from a potential-field source-surface (PFSS) extrapolation of magnetograph measurements from the Mount Wilson Observatory (MWO). As indicated by the 
yellow dot, the filament eruption occurred along the PIL encircling the negative-polarity north polar cap. In order to account for the pair of transient coronal holes, most of the overlying coronal loops rooted between the polar hole boundary and the field-line "part" or separatrix on the equatorward side of the PIL must have opened up during the eruption. From the fact that the post-eruption arcade started to form well before the twin dimmings reached their maximum strength, we deduce that the innermost loops near the PIL pinched off while the mass loss was still at an early stage. This can be seen from the off-limb $19.5 \mathrm{~nm}$ ratio images in Figure 1, At 04:05 UT the pinch-off has already occurred, while the evacuation of mass is still ongoing as evidenced by the darker void at 06:05 UT.

It is often assumed that all of the entrained coronal material is expelled when the magnetic field opens up. That the background $17.1 \mathrm{~nm}$ emission undergoes relatively little change during filament eruptions provides strong evidence to the contrary: most of the cooler coronal plasma does not escape before the field closes down again but instead remains trapped and never leaves the Sun. This result is consistent with multi-wavelength Doppler observations of a transient coronal hole by Imada et al. (2007; see also Jin et al. 2009), using the EUV Imaging Spectrometer on Hinode. They found that the outflow speeds at the hole boundary were strongly temperature-dependent, with a steep transition from slow to fast flows occurring near $1 \mathrm{MK}$. As indicated by their Figure 6 , the velocity $v$ varies from $\sim 30 \mathrm{~km} \mathrm{~s}^{-1}$ in Fe IX to $\sim 90 \mathrm{~km} \mathrm{~s}^{-1}$ in Fe XII to $\sim 160 \mathrm{~km} \mathrm{~s}^{-1}$ in Fe XV. Correspondingly, the timescale $\tau_{\text {esc }} \sim R_{\odot} / v$ for the plasma to travel a solar radius varies from $\sim 7 \mathrm{hr}$ in Fe IX to $\sim 2 \mathrm{hr}$ in $4 \mathrm{hr}$ Fe XII to only $\sim 1 \mathrm{hr}$ in Fe XV. Since the transient holes in the January 14 and December 27 events reached their greatest dimming level $\sim 3$ to $4 \mathrm{hr}$ after they first appeared in the base-ratio images, we conclude that the Fe IX emitting plasma did not have sufficient time to escape before the onset of reconnection.

An interesting analogy may be drawn between the temperature dependence of transient holes and the relationship between polar plumes and the interplume regions in coronal holes. EUV plumes, which are best observed in lower-temperature lines like Fe IX $17.1 \mathrm{~nm}$, are both denser and cooler than the interplume medium; moreover, Doppler measurements indicate that the flow speeds in plumes are much smaller (at low heights) than in the rest of the coronal hole (see e.g., Wilhelm et al. 1998; Cranmer et al. 1999). Evidently, when the magnetic field opens up during a filament eruption, the hotter component of the corona behaves like the interplume medium, whereas the cooler component behaves like the plume gas. According to this analogy, the hot and cool components would exist along separate field lines or "strands" of flux tubes.

As demonstrated by the events described in section 2, the $17.1 \mathrm{~nm}$ corona shows its most pronounced dimmings not during the eruption, but during the reconnection phase. These 
propagating dark ribbons or "heat waves" occur at the leading edge of the $17.1 \mathrm{~nm}$ posteruption arcade, and result from the reconnective heating of the cool plasma that was not ejected during the eruption. By implication, the post-eruption brightenings seen at 19.5 and $28.4 \mathrm{~nm}$ have two different sources: reconnected loops that were refilled via chromospheric evaporation and underwent subsequent cooling, and reconnected loops that were already filled with cool plasma, but were then heated to temperatures well above $1 \mathrm{MK}$.

\section{Conclusions}

Our main points may be summarized as follows:

1. When viewed either on the disk or at the limb, transient coronal holes are much less visible in Fe IX $17.1 \mathrm{~nm}$ than in higher-temperature emission lines such as Fe XII $19.5 \mathrm{~nm}$. This observational result implies that most of the cooler coronal plasma does not escape when the magnetic field opens up.

2. The cooler plasma remains trapped because it flows outward too slowly to escape before the field lines close down again. As shown by the Doppler measurements of Imada et al. (2007), the outflow velocities in transient holes decrease dramatically at temperatures below $\sim 1 \mathrm{MK}$, from close to the sound speed to only $\sim 30 \mathrm{~km} \mathrm{~s}^{-1}$.

3. The strongest darkenings in $17.1 \mathrm{~nm}$ occur not during the opening-up of the magnetic field, but when it closes down again. The trapped plasma is then heated to high temperatures, producing darkenings in $17.1 \mathrm{~nm}$ which coincide with brightenings in 28.4 and $19.5 \mathrm{~nm}$. As the post-eruption arcade expands, a dark wavefront is observed in $17.1 \mathrm{~nm}$ at the leading

edges of the arcade, which appears as an outward-propagating dark ribbon when viewed on the disk. Off-limb this gives the (wrong) impression of dark loops that are "opening up".

4. An analogy exists between the temperature dependence of transient holes and the relationship between polar plumes and the interplume regions in coronal holes, with the plume (interplume) behaving in some ways like the cool (hot) plasma in transient holes.

Many important questions remain to be addressed. What is the physical reason for the steep transition between slow and fast outflow near 1 MK? Is a greater fraction of the cool plasma ejected in energetic events involving fast CMEs? Are dark on-disk "heat waves" also seen in higher-temperature lines such as Fe XII $19.5 \mathrm{~nm}$, where the pinched-off loops are refilled by chromospheric evaporation? This preliminary study suggests that the continued investigation of temperature effects in coronal dimmings may provide a key to a better physical understanding of CME eruptions. 
We are indebted to G. A. Doschek, G. Stenborg, I. Ugarte-Urra, H. P. Warren and P. R. Young for informative discussions. The SECCHI data is produced by an international consortium of the NRL, LMSAL and NASA GSFC (USA), RAL and U. Bham (UK), MPS (Germany), CSL (Belgium), IOTA and IAS (France). This work was supported by NASA and the Office of Naval Research.

\section{REFERENCES}

Attrill, G., Nakwacki, M. S., Harra, L. K., van Driel-Gesztelyi, L., Mandrini, C. H., Dasso, S., \& Wang, J. 2006, Sol. Phys., 238, 117

Cranmer, S. R., et al. 1999, ApJ, 511, 481

Dai, Y., Auchère, F., Vial, J.-C., Tang, Y. H., \& Zong, W. G. 2010, ApJ, 708, 913

Del Zanna, G., Bromage, B. J. I., \& Mason, H. E. 2003, A\&A, 398, 743

Harra, L. K., et al. 2007, PASJ, 59, S801

Harrison, R. A., Bryans, P., Simnett, G. M., \& Lyons, M. 2003, A\&A, 400, 1071

Harrison, R. A., \& Lyons, M. 2000, A\&A, 358, 1097

Howard, R. A., et al. 2008, Space Sci. Rev., 136, 67

Howard, R. F., \& Harvey, J. W., \& Forgach, S. 1990, Sol. Phys., 130, 295

Howard, T. A., \& Harrison, R. A. 2004, Sol. Phys., 219, 315

Imada, S., et al. 2007, PASJ, 59, S793

Jin, M., Ding, M. D., Chen, P. F., Fang, C., \& Imada, S. 2009, ApJ, 702, 27

Kahler, S. W., \& Hudson, H. S. 2001, J. Geophys. Res., 106, 29239

Kopp, R. A., \& Pneuman, G. W. 1976, Sol. Phys., 50, 85

Reinard, A. A., \& Biesecker, D. A. 2008, ApJ, 674, 576

Reinard, A. A., \& Biesecker, D. A. 2009, ApJ, 705, 914

Sheeley, N. R., Jr., Warren, H. P., \& Wang, Y.-M. 2004, ApJ, 616, 1224

Sheeley, N. R., Jr., Warren, H. P., \& Wang, Y.-M. 2007, ApJ, 671, 926

Warren, H. P., Bookbinder, J. A., Forbes, T. G., Golub, L., Hudson, H. S., Reeves, K., \& Warshall, A. 1999, ApJ, 527, L121 
Wilhelm, K., Marsch, E., Dwivedi, B. N., Hassler, D. M., Lemaire, P., Gabriel, A. H., \& Huber, M. C. E. 1998, ApJ, 500, 1023 
$17.1 \mathrm{~nm}$
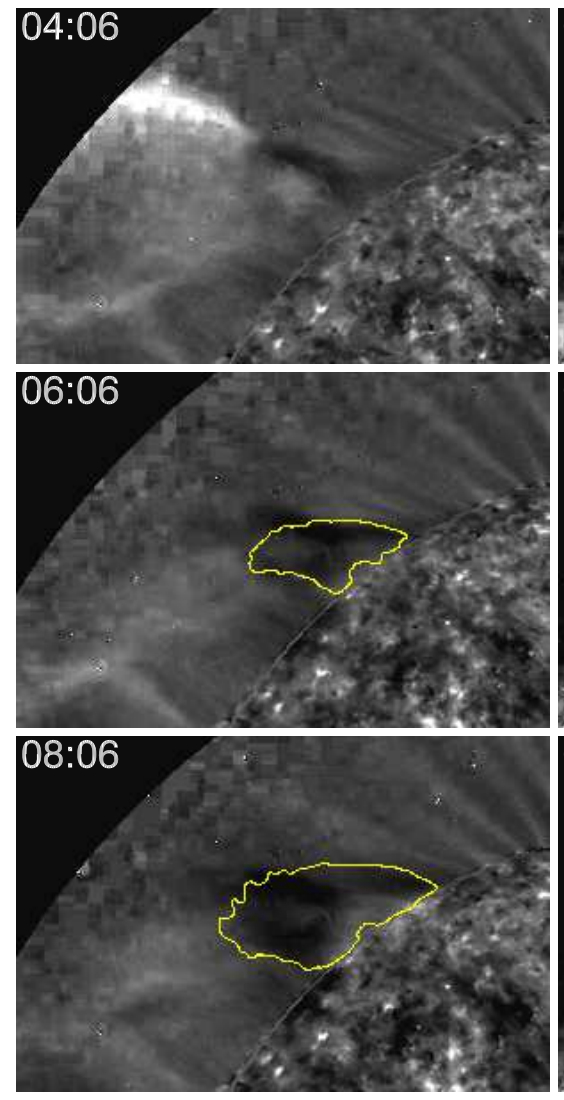

$19.5 \mathrm{~nm}$

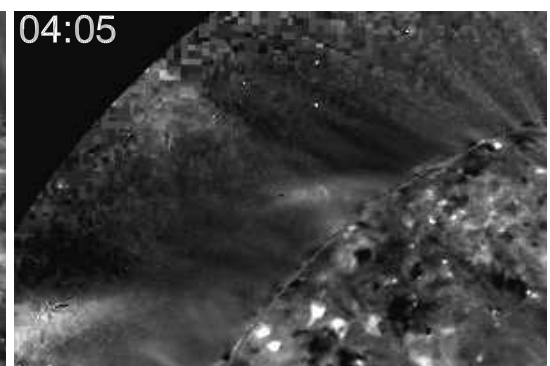

06:05

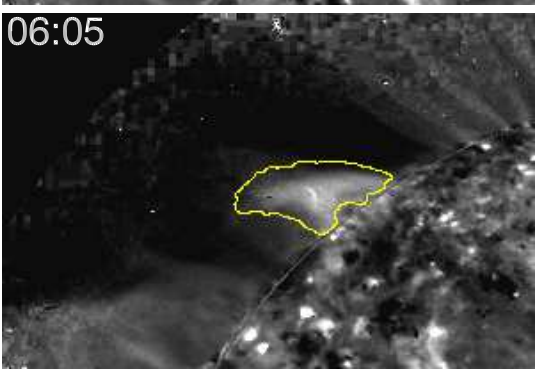

08:05
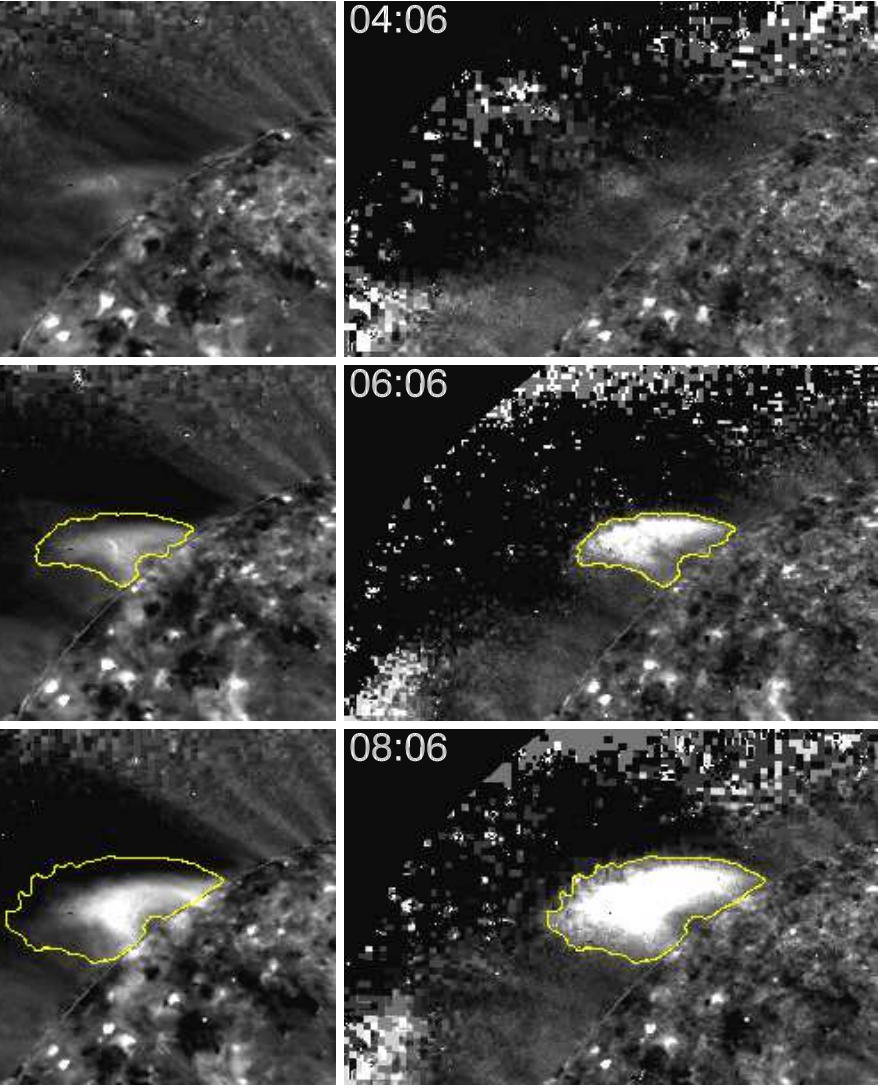

Fig. 1. - Eruption of a filament at the northeast limb on 2009 January 14, as viewed from STEREO/EUVI A. The images show the ratio of the local brightness at the indicated time relative to a base image taken at 19:06 UT on January 13, for each of the emission lines Fe IX $17.1 \mathrm{~nm}$ (left column), Fe XII $19.5 \mathrm{~nm}$ (middle column), and Fe XV $28.4 \mathrm{~nm}$ (right column). The on-disk areas of these base-ratio images have been corrected for solar differential rotation. In the two hotter lines (19.5 and $28.4 \mathrm{~nm})$, the dimming appears as a dark void above the limb; this void is not seen in the cooler $17.1 \mathrm{~nm}$ bandpass. The $17.1 \mathrm{~nm}$ images at 06:06 and 08:06 UT show dark features that coincide with the outer edges (indicated by the yellow contours) of the bright post-eruptive loops in $28.4 \mathrm{~nm}$. [A movie is available online.] 
$17.1 \mathrm{~nm}$
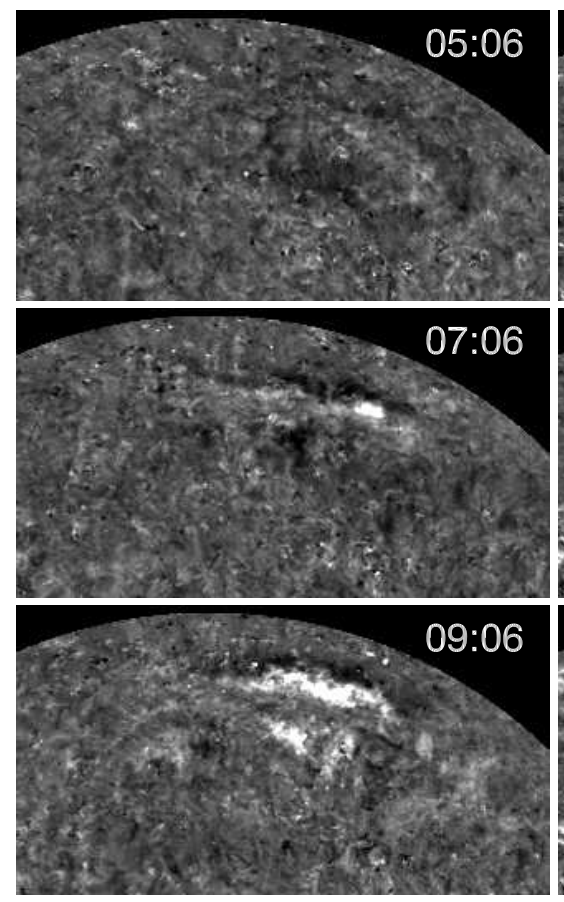

$19.5 \mathrm{~nm}$
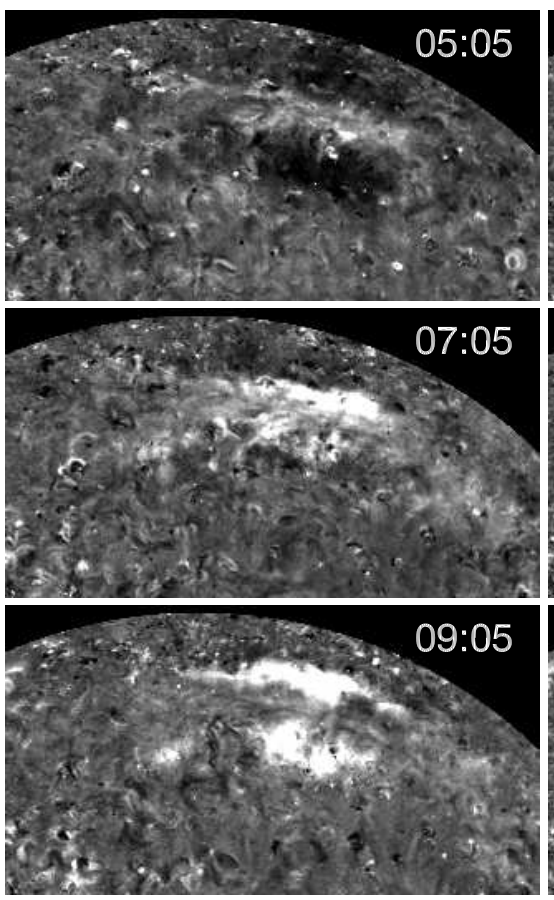

$28.4 \mathrm{~nm}$
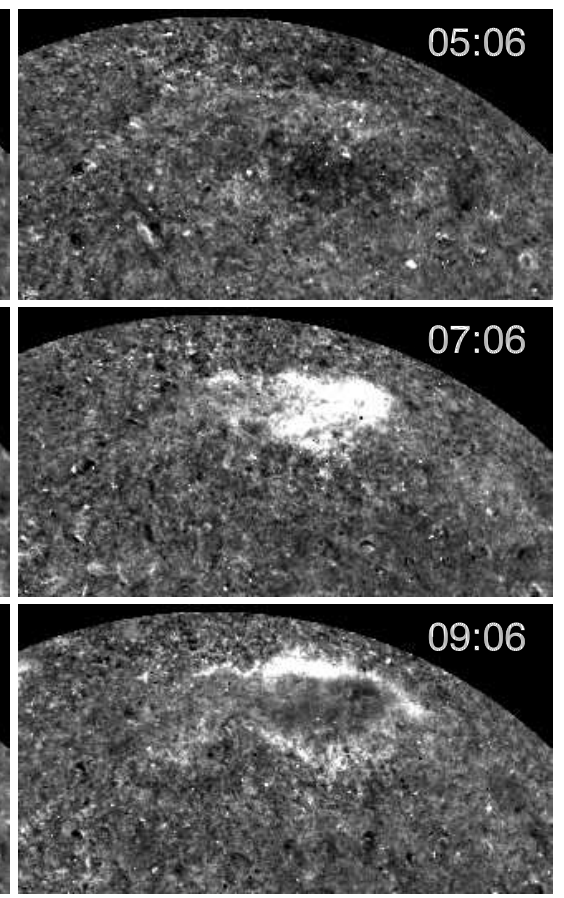

Fig. 2. - The filament eruption of 2009 January 14, as viewed on the disk from STEREO/EUVI B (compare the limb view of Figure 1). The images show the ratio of the local brightness at the indicated time relative to that recorded $2 \mathrm{hr}$ earlier, after removing the effect of solar rotation. The "real" dimming is best observed in Fe XII $19.5 \mathrm{~nm}$ at 05:05 UT; it is less pronounced in the $28.4 \mathrm{~nm}$ bandpass, which contains contributions from cooler lines like Si VII and Mg7, and is barely visible in Fe IX 17.1 nm. At 07:06 and 09:06 UT, the $17.1 \mathrm{~nm}$ running-ratio images show a dark "heat wave" which coincides with the brightenings in 28.4 and $19.5 \mathrm{~nm}$. [A movie is available online.] 

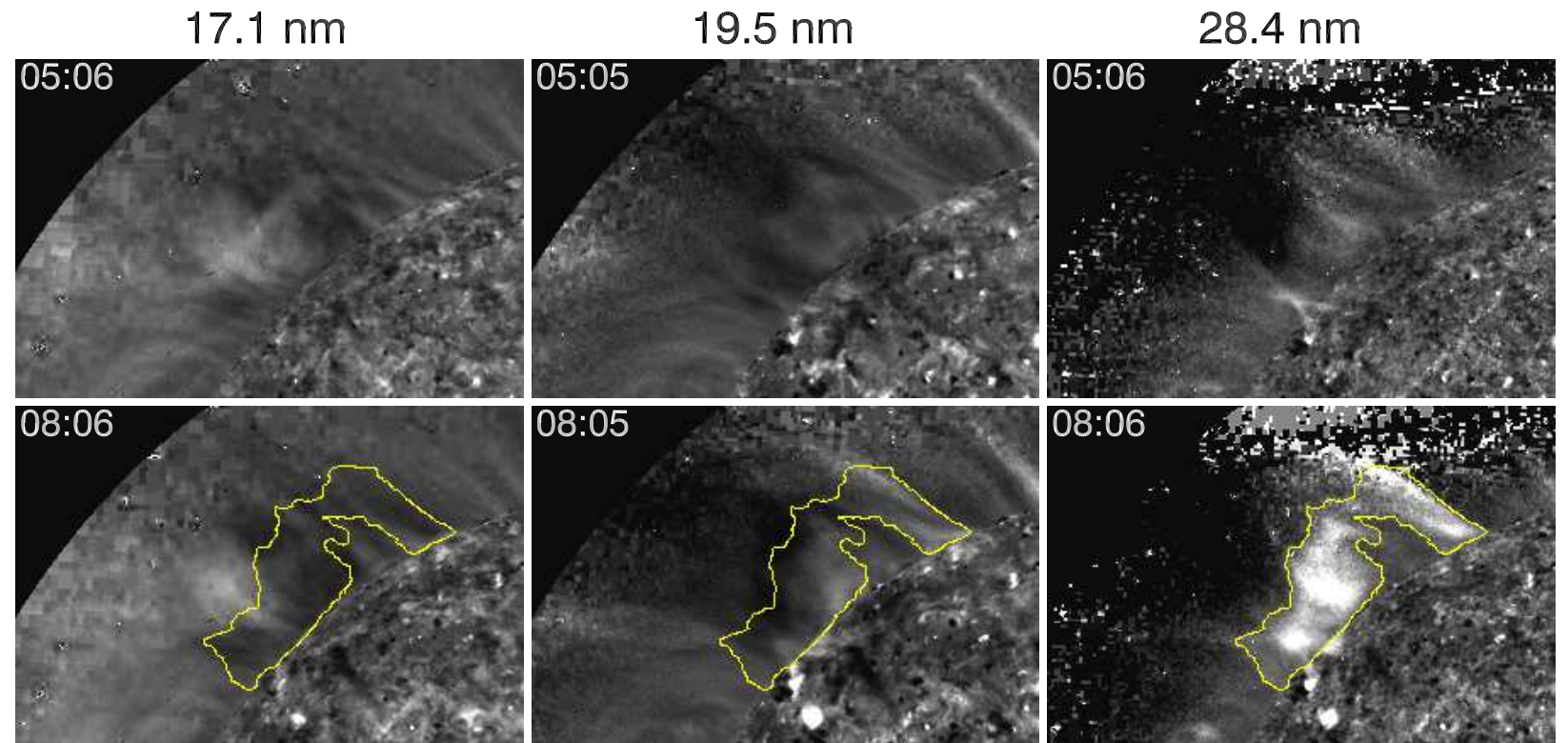

\section{8:05}

\section{8:06}
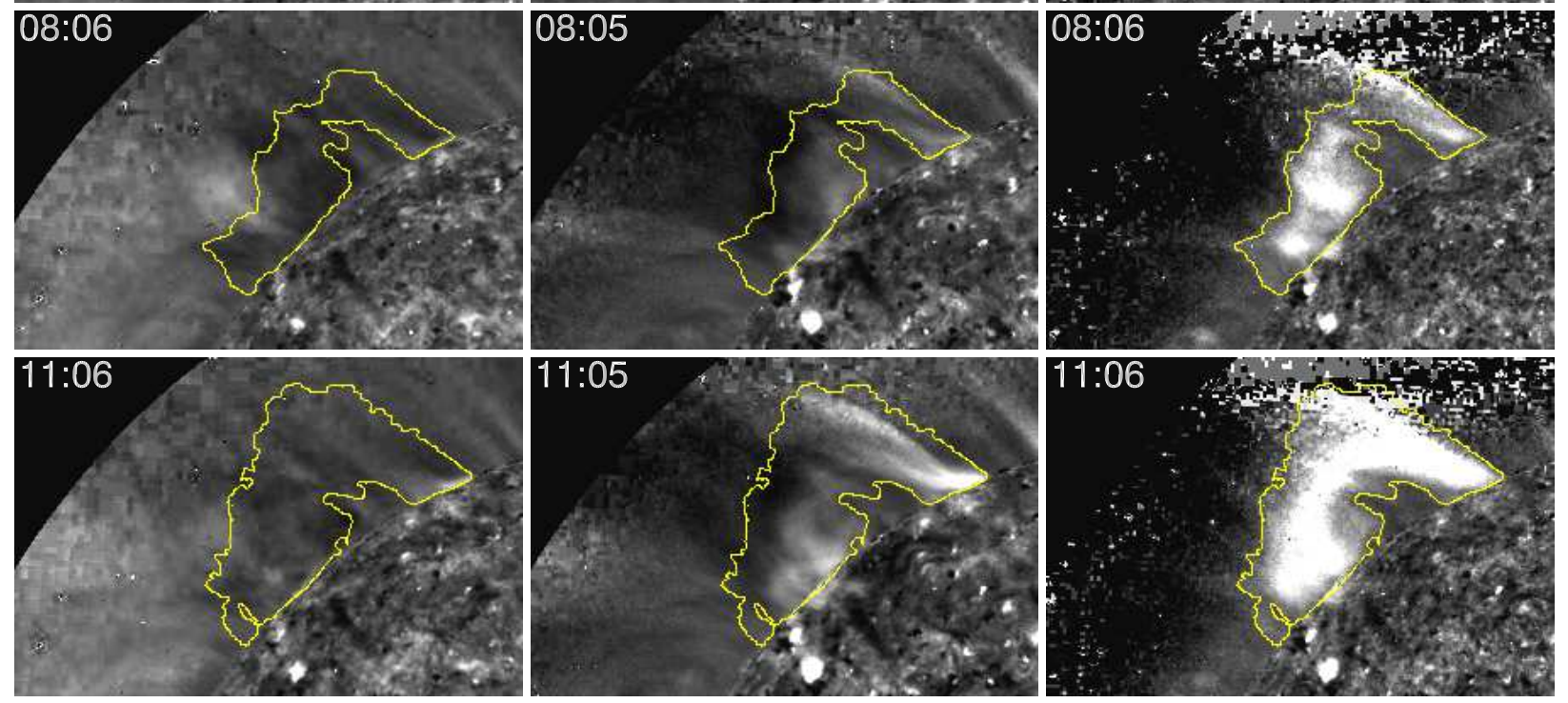

Fig. 3.- Filament eruption at the northeast limb on 2008 December 27, as viewed from EUVI A. The images show the ratio of local brightness at the indicated time relative to a base image taken at 23:06 UT on December 26, after removing the effect of solar differential rotation from the on-disk area. Yellow contours indicate the boundaries of the bright $28.4 \mathrm{~nm}$ post-eruption arcade. [A movie is available online.] 
$17.1 \mathrm{~nm}$
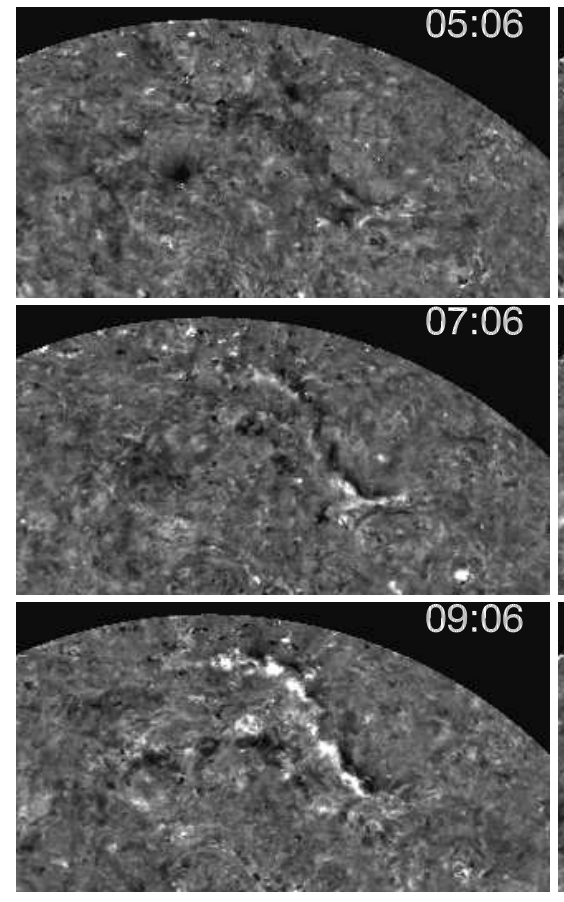

$19.5 \mathrm{~nm}$
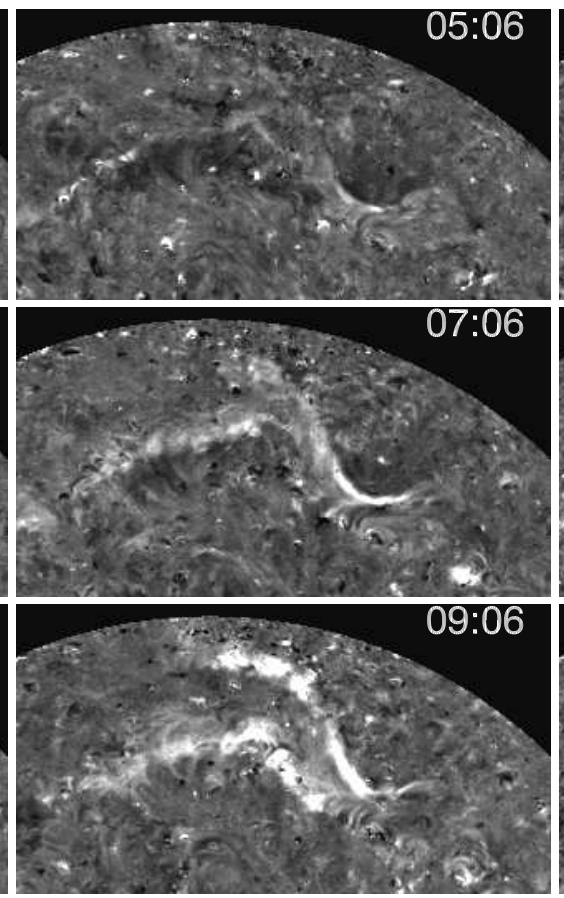

$28.4 \mathrm{~nm}$
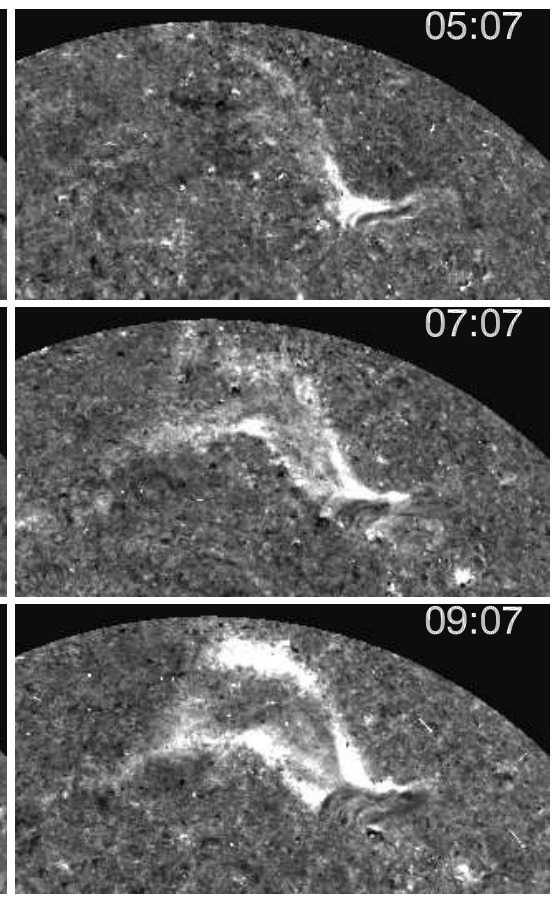

Fig. 4.- The filament eruption of 2008 December 27, as viewed on the disk from EUVI B (compare the limb view of Figure 3). The images show the ratio of the local brightness at the indicated time relative to that recorded $2 \mathrm{hr}$ earlier, after correcting for solar rotation. The "real" dimming is easiest to see in the $19.5 \mathrm{~nm}$ bandpass. The $17.1 \mathrm{~nm}$ running-ratio images show a dark, outward-propagating "heat wave". [A movie is available online.] 


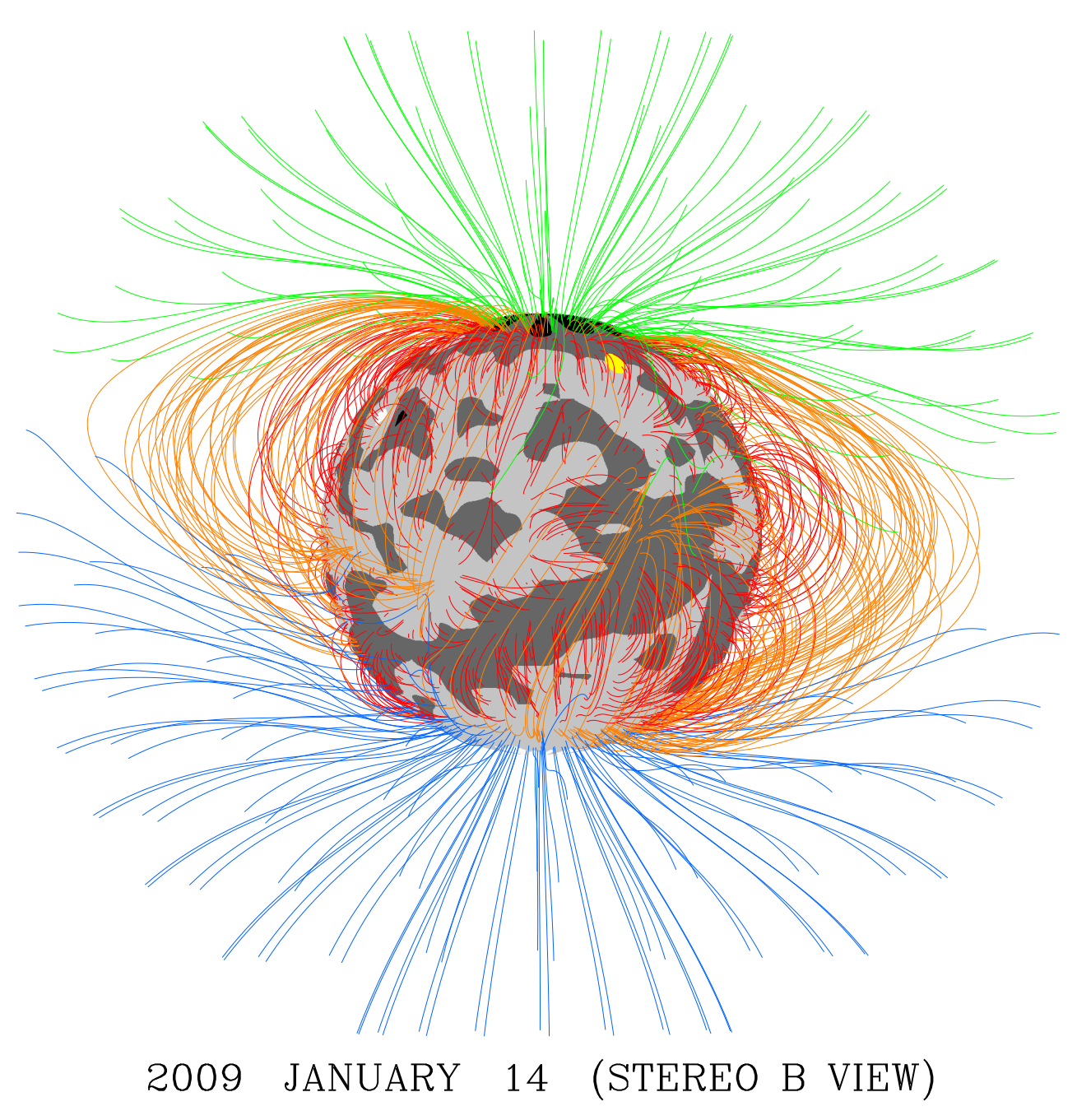

Fig. 5.- Coronal magnetic field on 2009 January 14, as viewed from STEREO B. The field lines were derived from a PFSS extrapolation of the MWO photospheric measurements for Carrington rotation 2079; the source surface was taken to be at a heliocentric distance of $r=2.5 R_{\odot}$. The yellow dot marks the approximate location of the filament eruption in Figure 2. Closed loops are coded orange if they extend beyond $r=1.5 R_{\odot}$, red otherwise; open field lines are blue (green) if they have positive (negative) polarity. Black, dark gray, light gray, and white denote areas of the photosphere where the radial field component lies in the ranges $B_{r}<-6 \mathrm{G},-6 \mathrm{G}<B_{r}<0 \mathrm{G}, 0 \mathrm{G}<B_{r}<+6 \mathrm{G}$, and $B_{r}>+6 \mathrm{G}$, respectively. 